\title{
Maximal Violation of Bell's Inequalities is Generic in Quantum Field Theory
}

\author{
Stephen J. Summers ${ }^{1}$ and Reinhard Werner ${ }^{2}$ \\ ${ }^{1}$ Department of Mathematics, University of Rochester, Rochester, NY 14627, USA \\ ${ }^{2}$ Fachbereich Physik, Universität Osnabrück, D-4500 Osnabrück, Federal Republic of Germany
}

\begin{abstract}
Under weak technical assumptions on a net of local von Neumann algebras $\{\mathscr{A}(\mathcal{O})\}$ in a Hilbert space $\mathscr{H}$, which are fulfilled by any net associated to a quantum field satisfying the standard axioms, it is shown that for every vector state $\phi$ in $\mathscr{H}$ there exist observables localized in complementary wedgeshaped regions in Minkowski space-time that maximally violate Bell's inequalities in the state $\phi$. If, in addition, the algebras corresponding to wedgeshaped regions are injective (which is known to be true in many examples), then the maximal violation occurs in any state $\phi$ on $\mathscr{B}(\mathscr{H})$ given by a density matrix.
\end{abstract}

\section{Introduction}

With the discovery of Bell's inequalities $[6,7]$ and their subsequent refinements $[10,24,26,32]$, it became possible for the first time to test experimentally whether or not certain concepts of classical physics can be used in a description (at least within a fairly general metatheoretic framework) of processes that take place on an atomic or subatomic scale. The inequalities concern statistical correlations of measurements made on two parts of one system and are derived from two basic assumptions. One is that all measured correlations $p(\alpha, \beta)$ between outcomes $\alpha$ measured on one subsystem and outcomes $\beta$ measured on the other subsystem can be modelled within a classical probabilistic theory (which subsumes, of course, deterministic theories). The second is that this description is "local" in the sense that the choice of a measuring device operating upon one part of the system does not affect the probabilities of outcomes measured on the other part. For a more thorough discussion of the assumptions and the metatheoretical framework within which Bell's inequalities can be derived, see [32].

It is not our intent to enter into a philosophical discussion of Bell's inequalities in this forum. Our aim is to show that, just as quantum mechanics does, quantum field theory (QFT) predicts a maximal violation of Bell's inequalities, and that in fact, unlike quantum mechanics, QFT predicts that this maximal violation is generic in a sense we shall explain. 
Let $\{\mathscr{A}(\mathcal{O})\}_{\mathcal{O} \subset \mathbb{R}^{4}}$ be a net of local algebras satisfying the usual axioms $[27,1]$ and let $\phi$ be a state on the algebra $\mathscr{A}$ of quasilocal observables generated by $\{\mathscr{A}(\mathcal{O})\}$. Then for $\mathcal{O}_{1}, \mathcal{O}_{2}$ two spacelike separated open regions in Minkowski space-time, we define the maximal Bell correlation in the state $\phi$ to be:

$$
\beta\left(\phi, \mathscr{A}\left(\mathcal{O}_{1}\right), \mathscr{A}\left(\mathcal{O}_{2}\right)\right) \equiv \frac{1}{2} \sup \phi\left(A_{1}\left(B_{1}+B_{2}\right)+A_{2}\left(B_{1}-B_{2}\right)\right),
$$

where the supremum is taken over all selfadjoint $A_{i} \in \mathscr{A}\left(\mathcal{O}_{1}\right), B_{j} \in \mathscr{A}\left(\mathcal{O}_{2}\right)$, satisfying $-1 \leqq A_{i} \leqq 1,-1 \leqq B_{j} \leqq 1, i, j=1,2$. Such quadruples $\left\{A_{i}, B_{j}\right\}$ are henceforth called admissible.) It was shown in [32] that the Clauser-Horne version of Bell's inequalities [10] can be written in algebraic QFT as:

$$
\beta\left(\phi, \mathscr{A}\left(\mathcal{O}_{1}\right), \mathscr{A}\left(\mathcal{O}_{2}\right)\right)=1 \text {. }
$$

It is known [32] that the following inequality must hold a priori in algebraic QFT for any state $\phi$ and regions $\mathcal{O}_{1}, \mathcal{O}_{2}$ as above:

$$
\beta\left(\phi, \mathscr{A}\left(\mathcal{O}_{1}\right), \mathscr{A}\left(\mathcal{O}_{2}\right)\right) \leqq \sqrt{2} .
$$

If equality is attained in (1.3), then we say that Bell's inequalities are maximally violated [in the state $\phi$ by suitable observables in $\mathscr{A}\left(\mathcal{O}_{1}\right), \mathscr{A}\left(\mathcal{O}_{2}\right)$ ]. In hindsight, it is seen that quantum mechanics predicts the attainment of $\sqrt{2}$ for the maximal Bell correlation for suitable states and observables $[10,4]$, and that the experiments reported in [3-5] yield this maximal violation of $\sqrt{2}$, within experimental error.

In [33] we showed that if $\phi_{0}$ is the vacuum state of a free Bose or Fermi field theory and $\mathscr{A}(W), \mathscr{A}\left(W^{\prime}\right)$ are the corresponding observable algebras for the wedge regions $W$ and $W^{\prime}\left(W^{\prime}\right.$ is the causal complement of $W$ ), then

$$
\beta\left(\phi_{0}, \mathscr{A}(W), \mathscr{A}\left(W^{\prime}\right)\right)=\sqrt{2} .
$$

For dilatation-invariant, free field theories, (1.4) has been demonstrated to hold for other space-time regions $\mathcal{O}_{1}, \mathcal{O}_{2}$ (including bounded ones) [32,33]. Thus, even in free field theories the vacuum fluctuations are such that suitable observables maximally violate Bell's inequalities. The proof of these results pointed to a connection with the modular theory of the von Neumann algebras of the net $\{\mathscr{A}(\mathcal{O})\}$, and investigation of this connection soon made it clear that much stronger theorems can be proven by invoking deep results of Connes, Størmer, Driessler and others.

Specifically (and the assumptions will be made explicit in Sect. 3), we shall show that for any net of local von Neumann algebras $\{\mathscr{A}(\mathcal{O})\}$ on a Hilbert space $\mathscr{H}$ and any vector state $\phi$ on $\mathscr{B}(\mathscr{H})$,

$$
\beta\left(\phi, \mathscr{A}(W), \mathscr{A}(W)^{\prime}\right)=\sqrt{2}
$$

[ $\mathscr{A}(W)^{\prime}$ is the commutant of $\mathscr{A}(W)$ and $W$ is any wedge-shaped region]. Thus, if the net satisfies duality for the wedge regions, then

$$
\beta\left(\phi, \mathscr{A}(W), \mathscr{A}\left(W^{\prime}\right)\right)=\sqrt{2} .
$$

Moreover, if the wedge algebras are injective (which is known to be the case in many examples), then for any normal state $\phi$ on $\mathscr{B}(\mathscr{H}),(1.5)$ holds, and thus (1.6) 
obtains if duality is satisfied. This is what we mean by the genericity of maximal violation - in (nearly) every quantum field theory, and for every normal state in such a theory, there are observables localized in $W$ and $W^{\prime}$ that maximally violate Bell's inequalities. Surprisingly, this genericity of maximal violation is connected with a technical property from the theory of operator algebras that was originally identified for totally different reasons. We shall show, in fact, that (1.5) holds for all normal $\phi$ on $\mathscr{B}(\mathscr{H})$ if and only if it is possible to find a sequence of admissible quadruples that is a maximal violator for all normal states, and that this holds if and only if the wedge algebras $\mathscr{A}(W)$ are strongly stable in the sense of [34]. All injective, infinite factors (excepting some type $\mathrm{III}_{0}$ ) are strongly stable $[14,2]$.

We proceed now to the details. It should be mentioned that all results hold for two, three or four space-time dimensions.

\section{Results on von Neumann Algebras}

We commence by proving a result about type III $_{1}$ von Neumann algebras (see [11] for the classification of type III factors), which we shall apply to QFT in the following section. $\mathscr{B}(\mathscr{H})$ will always denote the von Neumann algebras of all bounded operators on the Hilbert space $\mathscr{H}$.

Theorem 2.1. Let $\mathscr{M}$ be a type $I I I_{1}$ factor with cyclic and separating vector in a Hilbert space $\mathscr{H}$ and let $\phi$ be a vector state on $\mathscr{B}(\mathscr{H})$. Then $\beta\left(\phi, \mathscr{M}, \mathscr{M}^{\prime}\right)=\sqrt{2}$.

Proof. 1. First it is shown that $\beta\left(\phi, \mathscr{M}, \mathscr{M}^{\prime}\right)$ is independent of the choice of vector state $\phi$. Let $\Omega_{1}, \Omega_{2}$ be unit vectors in $\mathscr{H}$ and $\phi_{i}(A) \equiv\left\langle\Omega_{i}, A \Omega_{i}\right\rangle, A \in \mathscr{B}(\mathscr{H})$, the corresponding vector states on $\mathscr{B}(\mathscr{H})$. By the definition of $\beta$ there are, for any $\varepsilon_{1}>0$, selfadjoint contractions $A_{i} \in \mathscr{M}, B_{i} \in \mathscr{M}^{\prime}(i=1,2)$ such that

$$
\frac{1}{2}\left\langle\Omega_{1},\left(A_{1}\left(B_{1}+B_{2}\right)+A_{2}\left(B_{1}-B_{2}\right)\right) \Omega_{1}\right\rangle \geqq \beta\left(\phi_{1}, \mathscr{M}, \mathscr{M}^{\prime}\right)-\varepsilon_{1} .
$$

By Corollary 6 in [15] there are, for any $\varepsilon_{2}>0$, unitaries $U \in \mathscr{M}, U^{\prime} \in \mathscr{M}^{\prime}$ such that $\left\|\Omega_{2}-U U^{\prime} \Omega_{1}\right\| \leqq \varepsilon_{2}$. Thus, with $\widetilde{A}_{i}=U A_{i} U^{*} \in \mathscr{M}, \widetilde{B}_{i}=U^{\prime} B_{i} U^{*} \in \mathscr{M}^{\prime},\left\{\widetilde{A}_{i}, \widetilde{B}_{j}\right\}_{i, j=1,2}$ is an admissible quadruple and one has

$$
\begin{aligned}
\beta\left(\phi_{2}, \mathscr{M}, \mathscr{M}^{\prime}\right) & \geqq \frac{1}{2}\left\langle\Omega_{2},\left(\tilde{A}_{1}\left(\widetilde{B}_{1}+\widetilde{B}_{2}\right)+\tilde{A}_{2}\left(\widetilde{B}_{1}-\widetilde{B}_{2}\right)\right) \Omega_{2}\right\rangle \\
& \geqq \beta\left(\phi_{1}, \mathscr{M}, \mathscr{M}^{\prime}\right)-\varepsilon_{1}-O\left(\varepsilon_{2}\right) .
\end{aligned}
$$

Hence, by symmetry between $\phi_{1}$ and $\phi_{2}, \beta\left(\phi_{1}, \mathscr{M}, \mathscr{M}^{\prime}\right)=\beta\left(\phi_{2}, \mathscr{M}, \mathscr{M}^{\prime}\right)$.

2. It remains to be proven that $\beta\left(\phi, \mathscr{M}, \mathscr{M}^{\prime}\right)=\sqrt{2}$ holds for a suitable vector state $\phi(\cdot)=\langle\Omega, \cdot \Omega\rangle, \Omega \in \mathscr{H}$. Consider a faithful normal state $\phi_{1} \in \mathscr{M}_{*}$ of the form $\phi_{1} \simeq \phi_{0} \otimes \frac{1}{2}$ tr with respect to some isomorphism $\mathscr{M} \simeq \mathscr{M} \otimes M_{2}(\mathbb{C})$, where $M_{2}(\mathbb{C})$ is the algebra of all $2 \times 2$ matrices with complex entries, and consider the isometry $v \simeq 1 \otimes\left(\begin{array}{ll}0 & 1 \\ 0 & 0\end{array}\right)$. Then $v$ is in the centralizer of $\mathscr{M}$ in $\phi_{1}$ and for any $\alpha \in \mathbb{R}, A(\alpha)$ $\equiv \exp (i \alpha) v+\exp (-i \alpha) v^{*}$ is selfadjoint and unitary. Let $\Omega \in \mathscr{H}$ be a vector with $\phi_{1}(A)=\langle\Omega, A \Omega\rangle$ for all $A \in \mathscr{M}$ (the existence of this vector is assured by Theorem 2.7.9 in [29]), and let $P^{\prime} \in \mathscr{M}^{\prime}$ be the projection onto $\mathscr{M} \Omega$. Since the operators $B_{i}$ yet to be chosen will be contained in $P^{\prime} \mathscr{M}^{\prime} P^{\prime}$, it can be assumed that 
$P^{\prime}=1$, i.e. that $\Omega$ is cyclic and separating for $\mathscr{M}$ (in other words, if $\Omega$ is not cyclic for $\mathscr{M}$, then cutting the Hilbert space $\mathscr{H}$ and the commutant $\mathscr{M}^{\prime}$ down to $P^{\prime} \mathscr{H}$ and $P^{\prime} \mathscr{M}^{\prime} P^{\prime}$ gives a lower bound on the original $\beta$; if this lower bound is $\sqrt{2}$, then the proof is completed).

Let $S=J \Delta^{1 / 2}$ be the modular involution associated with $(\mathscr{M}, \Omega)$. Then

$$
\begin{aligned}
\left\langle\Omega, A\left(\alpha_{1}\right) J A\left(\alpha_{2}\right) J \Omega\right\rangle & =\left\langle\Omega, A\left(\alpha_{1}\right) \Delta^{1 / 2} A\left(\alpha_{2}\right) \Omega\right\rangle \\
\text { (since } A\left(\alpha_{2}\right)=A\left(\alpha_{2}\right)^{*} \text { and } J A\left(\alpha_{2}\right) J \Omega & \left.=\Delta^{1 / 2} A\left(\alpha_{2}\right)^{*} \Omega\right) \\
& =\left\langle\Omega, A\left(\alpha_{1}\right) A\left(\alpha_{2}\right) \Omega\right\rangle=\cos \left(\alpha_{1}-\alpha_{2}\right),
\end{aligned}
$$

since $A\left(\alpha_{2}\right)$ is in the centralizer of $\mathscr{M}$ in $\phi_{1}$ and thus commutes with $\Delta^{1 / 2}$. Therefore, defining selfadjoint unitaries $A_{i} \in \mathscr{M}, B_{i} \in \mathscr{M}^{\prime}(i=1,2)$ by $A_{1}=A(0), A_{2}=A(\pi / 2)$, $B_{1}=J A(\pi / 4) J, B_{2}=J A(-\pi / 4) J$, one obtains

$$
\frac{1}{2}\left\langle\Omega,\left(A_{1}\left(B_{1}+B_{2}\right)+A_{2}\left(B_{1}-B_{2}\right)\right) \Omega\right\rangle=2 \cos \frac{\pi}{4}=\sqrt{2} .
$$

Remarks. 1. If $\mathscr{H}$ is a separable Hilbert space, as is always the case in QFT, then any type III algebra always has a cyclic and separating vector in $\mathscr{H}$ (Corollary 2.9.28 in [29]).

2. We anticipate that if $\mathscr{M}$ is any von Neumann algebra with no type I summand, then $\beta\left(\phi, \mathscr{M}, \mathscr{M}^{\prime}\right)=\sqrt{2}$ for any state $\phi$ determined by a vector that is cyclic and separating for $\mathscr{M}$. This has been verified for type II algebras, but we shall not present the proof here since the result probably has no relevance to QFT.

We note that Theorem 2.1 gives $\beta\left(\phi, \mathscr{M}, \mathscr{M}^{\prime}\right)=\sqrt{2}$ only for vector states $\phi$. In the theorem to be shown next, we give necessary and sufficient conditions on $\mathscr{M}$ in the Hilbert space $\mathscr{H}$ so that $\beta\left(\phi, \mathscr{M}, \mathscr{M}^{\prime}\right)=\sqrt{2}$ for every normal state $\phi$ on $\mathscr{B}(\mathscr{H})$, i.e. for every state given by a density matrix in $\mathscr{B}(\mathscr{H})$.

In Theorem 2.4(3) in [31] we showed that if $A_{i} \in \mathscr{M}$ and $B_{i} \in \mathscr{M}^{\prime}$ are admissible and satisfy

$$
\frac{1}{2} \phi\left(A_{1}\left(B_{1}+B_{2}\right)+A_{2}\left(B_{1}-B_{2}\right)\right)=\sqrt{2},
$$

for some given state $\phi$, then $A_{1}, A_{2}$ (respectively $B_{1}, B_{2}$ ) are in the centralizer of $\mathscr{M}$ (respectively $\mathscr{M}^{\prime}$ ) with respect to the state $\phi$. Any sequences $\left\{A_{i, \alpha}\right\}_{\alpha \in \mathbb{N}},\left\{B_{i, \alpha}\right\}_{\alpha \in \mathbb{N}}$, $i=1,2$, of admissible quadruples whose expectations in the left-hand side of (2.1) approach $\sqrt{2}$ would then be expected to be centralizing sequences in the state $\phi$. As it turns out (see below), if $\beta\left(\phi, \mathscr{M}, \mathscr{M}^{\prime}\right)=\sqrt{2}$ for all $\phi \in \mathscr{B}(\mathscr{H})_{*, 1}^{(+)}$, then there exists a sequence of admissible quadruples that constitutes four centralizing sequences in the sense of Dixmier and Lance. We remind the reader of the necessary background.

Definition. A bounded sequence $\left\{A_{n}\right\}_{n \in \mathbb{N}}$ in a separable factor $\mathscr{M}$ is called centralizing if

$$
\left|\phi\left(A_{n} A-A A_{n}\right)\right| \leqq \varepsilon_{n}\|A\|, \quad \text { all } A \in \mathscr{M}, \phi \in \mathscr{M}_{*},
$$

with $\varepsilon_{n} \rightarrow 0$ as $n \rightarrow \infty\left(\left\{\varepsilon_{n}\right\}_{n \in \mathbb{N}}\right.$ can depend on $\left.\phi\right)$. Two sequences $\left\{A_{n}\right\},\left\{B_{n}\right\}$ are said to be equivalent and are written $\left\{A_{n}\right\} \sim\left\{B_{n}\right\}$ if $\left\{A_{n}-B_{n}\right\}_{n \in \mathbb{N}}$ converges $\sigma^{*}$-strongly in $\mathscr{M}$. A centralizing sequence is called trivial if it is equivalent to a sequence of 
multiples of the identity. For a fixed ultrafilter $\omega$ on $\mathbb{N}$, a bounded sequence $\left\{A_{n}\right\}_{n \in \mathbb{N}}$ is called $\omega$-centralizing if $\varepsilon_{n} \rightarrow 0$ along the filter $\omega$. Let $\mathscr{C}(\mathscr{M})$ [respectively $\left.\mathscr{C}_{\omega}(\mathscr{M})\right]$ be the set of all centralizing (respectively $\omega$-centralizing) sequences of $\mathscr{M}$.

$\mathscr{C}_{\omega}(\mathscr{M})$ is a $C^{*}$-algebra, and we consider the subset $\mathscr{I}_{\omega}(\mathscr{M})$ of all sequences converging to zero $\sigma^{*}$-strongly. $\mathscr{I}_{\omega}(\mathscr{M})$ is a norm-closed ideal of $\mathscr{C}_{\omega}(\mathscr{M})[13,12]$. Let $\mathscr{M}_{\omega}(\mathscr{M}) \equiv \mathscr{C}_{\omega}(\mathscr{M}) / \mathscr{I}_{\omega}(\mathscr{M})$. Then $\mathscr{M}_{\omega}(\mathscr{M})$ is a finite von Neumann algebra with the trace $\tau_{\omega}$ defined by

$$
\tau_{\omega}(X)=\lim _{n \rightarrow \omega} \phi\left(A_{n}\right), \phi \in \mathscr{M}_{*, 1}^{(+)}
$$

where $X=\pi_{\omega}\left(\left\{A_{n}\right\}\right)$ with $\pi_{\omega}$ the canonical quotient map of $\mathscr{C}_{\omega}$ onto $\mathscr{M}_{\omega} \cdot \tau_{\omega}(X)$ does not depend on $\phi \in \mathscr{M}_{*, 1}^{(+)}[13,12]$.

We shall need the following result from the literature. $\mathscr{R}_{1}$ is the hyperfinite type $\mathrm{II}_{1}$ factor, and $\mathscr{A} \simeq \mathscr{B}$ denotes $\mathscr{A}$ is $W^{*}$-isomorphic to $\mathscr{B}$.

Theorem 2.2 (Theorem 2.2.1 in [13]). For a factor with a separable predual, the following conditions are equivalent.

(i) $\mathscr{M} \simeq \mathscr{M} \otimes \mathscr{R}_{1}$.

(ii) $\mathscr{M}_{\omega}(\mathscr{M})$ is not abelian for some ultrafilter $\omega$.

(iii) $\mathscr{M}_{\omega}(\mathscr{M})$ is a von Neumann algebra of type $I_{1}$.

A factor $\mathscr{M}$ is called strongly stable if any of the above conditions hold [34]. $\mathscr{T}(\mathscr{H})$ will denote the space of trace-class operators in $\mathscr{B}(\mathscr{H})$.

Theorem 2.3. Let $\mathscr{M}$ be a von Neumann algebra in a separable Hilbert space $\mathscr{H}$ with cyclic and separating vector. Then the following conditions are equivalent.

(i) $\mathscr{M} \simeq \mathscr{M} \otimes \mathscr{R}_{1}$.

(ii) For every normal state $\phi$ on $\mathscr{B}(\mathscr{H}), \beta\left(\phi, \mathscr{M}, \mathscr{M}^{\prime}\right)=\sqrt{2}$.

(iii) There exist sequences $\left\{A_{1, \alpha}\right\}_{\alpha \in \mathbb{N}},\left\{A_{2, \alpha}\right\}_{\alpha \in \mathbb{N}} \subset \mathscr{M},\left\{B_{1, \alpha}\right\}_{\alpha \in \mathbb{N}},\left\{B_{2, \alpha}\right\}_{\alpha \in \mathbb{N}} \subset \mathscr{M}^{\prime}$ of selfadjoint contractions such that $T_{\alpha} \equiv \frac{1}{2}\left(A_{1, \alpha}\left(B_{1, \alpha}+B_{2, \alpha}\right)+A_{2, \alpha}\left(B_{1, \alpha}-B_{2, \alpha}\right)\right)$ converges to $\sqrt{2} \cdot 1$ in the $\sigma(\mathscr{B}(\mathscr{H}), \mathscr{T}(\mathscr{H}))$-topology (i.e. the $\sigma$-weak operator topology) as $\alpha \rightarrow \infty$, where 1 is the identity on $\mathscr{H}$.

Proof. 1. The first step is to show that when $\mathscr{M}=\mathscr{R}_{1}$, then (iii) must hold. Let $\tilde{\mathscr{H}}=\bigotimes_{\alpha \in \mathbb{N}}\left(\mathscr{H}_{\alpha}, \Omega_{\alpha}\right)$ be the incomplete tensor product of $\mathscr{H}_{\alpha}=\mathbb{C}^{2} \otimes \mathbb{C}^{2}, \alpha \in \mathbb{N}$, with $\Omega_{\alpha}=\frac{1}{\sqrt{2}}\left(\Phi_{1} \otimes \Phi_{1}+\Phi_{2} \otimes \Phi_{2}\right) \equiv \Omega$ for some basis $\left\{\Phi_{1}, \Phi_{2}\right\} \in \mathbb{C}^{2}, \alpha \in \mathbb{N}$. Then by the construction of Araki and Woods [2], $\mathscr{R}_{1} \simeq \mathscr{R}\left(\mathscr{H}_{\alpha}, \mathscr{B}\left(\mathbb{C}^{2}\right) \otimes 1, \Omega_{\alpha}, \alpha \in \mathbb{N}\right)$ holds, using their notation for the factor on the right-hand side which is the infinite tensor product of $\mathscr{B}\left(\mathbb{C}^{2}\right) \otimes 1$ with itself on $\tilde{\mathscr{H}}$. Consider the following operators in $\mathscr{H}_{\alpha}$. Let $A_{1}, A_{2} \in \mathscr{B}\left(\mathbb{C}^{2}\right) \otimes 1$ and $B_{1}, B_{2} \in 1 \otimes \mathscr{B}\left(\mathbb{C}^{2}\right)$ be selfadjoint contractions with

$$
\langle\Omega, T \Omega\rangle \equiv\left\langle\Omega, \frac{1}{2}\left(A_{1}\left(B_{1}+B_{2}\right)+A_{2}\left(B_{1}-B_{2}\right)\right) \Omega\right\rangle=\sqrt{2} .
$$

From Theorem 2.4 of [32] it is known that such operators $A_{1}, A_{2}$ and $A_{3} \equiv \frac{i}{2}\left[A_{1}, A_{2}\right]\left(\right.$ respectively $B_{1}, B_{2}$ and $\left.B_{3} \equiv \frac{i}{2}\left[B_{1}, B_{2}\right]\right)$ must give a realization of the Pauli spin matrices. A straightforward calculation shows that (2.2) is in fact 
fulfilled by the choices described as follows. Let $\sigma_{1}, \sigma_{2}, \sigma_{3}$ be some realization of the Pauli spin matrices such that $\Phi_{1}$ (respectively $\Phi_{2}$ ) is the eigenstate of $\sigma_{3}$ corresponding to the eigenvalue +1 (respectively -1 ). If $\sigma$ is the matrix vector $\left(\sigma_{1}, \sigma_{2}, \sigma_{3}\right)$ and $\hat{a}\left(\theta_{i}\right), \hat{b}\left(\theta_{j}\right)$ coplanar unit vectors in $\mathbb{R}^{3}$ obtained by rotating a given unit vector $\hat{e}$ in the plane by the angle $\theta_{i}, \theta_{j}, i, j=1,2$, then define $A_{1}=\sigma \cdot \hat{a}(0) \otimes 1$, $A_{2}=\boldsymbol{\sigma} \cdot \hat{\mathbf{a}}(\pi / 2) \otimes 1, B_{1}=1 \otimes \boldsymbol{\sigma} \cdot \mathbf{b}(\pi / 4), B_{2}=1 \otimes \boldsymbol{\sigma} \cdot \hat{\mathbf{b}}(-\pi / 4)$. These operators determine an admissible quadruple that verifies (2.2).

Continuing with the proof, let $\widetilde{A}_{i, \alpha}, \widetilde{B}_{j, \alpha}, \widetilde{T}_{\alpha} \in \mathscr{B}(\tilde{\mathscr{H}})$ denote copies of the operators just constructed, acting in the $\alpha$-th tensor factor alone. Let furthermore $\Phi=\bigotimes_{\alpha \in \mathbb{N}} \Phi_{\alpha}, \Psi=\bigotimes_{\alpha \in \mathbb{N}} \Psi_{\alpha}$, with $\Phi_{\alpha}=\Psi_{\alpha}=\Omega_{\alpha}$ for almost all $\alpha$. By definition of the incomplete infinite tensor product, such vectors are total in $\tilde{\mathscr{H}}$. But one has $\left\langle\Phi, \widetilde{T}_{\alpha} \Psi\right\rangle=\langle\Phi, \Psi\rangle \cdot \sqrt{2}$ for all $\alpha$ larger than some $\alpha_{0}$ (depending on $\Phi$ and $\Psi$ ). Since the sequence $\left\{\widetilde{T}_{\alpha}\right\}_{\alpha \in \mathbb{N}}$ is norm-bounded, it follows that $\widetilde{T}_{\alpha} \rightarrow \sqrt{2} \cdot 1$ in the weak operator topology on $\mathscr{B}(\tilde{\mathscr{H}})$. Of course, $\tilde{A}_{i, \alpha} \in \mathscr{R}\left(\mathscr{H}_{\beta}, \mathscr{B}\left(\mathbb{C}^{2}\right) \otimes 1, \Omega_{\beta}, \beta \in \mathbb{N}\right)$ and $\widetilde{B}_{i, \alpha} \in \mathscr{R}\left(\mathscr{H}_{\beta}, 1 \otimes \mathscr{B}\left(\mathbb{C}^{2}\right), \Omega_{\beta}, \beta \in \mathbb{N}\right)$ for all $\alpha$, and $\bigotimes_{\alpha \in \mathbb{N}} \Omega_{\alpha}$ is cyclic and separating for these algebras. Since $\mathscr{M}$ has a cyclic and separating vector in $\mathscr{H}, \mathscr{M}=\mathscr{R}_{1}$ and $\mathscr{R}\left(\mathscr{H}_{\alpha}, \beta\left(\mathbb{C}^{2}\right) \otimes 1, \Omega_{\alpha}, \alpha \in \mathbb{N}\right)$ are unitarily equivalent with a unitary $W: \mathscr{H} \rightarrow \mathscr{\mathscr { H }}$ that also intertwines their commutants $\mathscr{R}_{1}^{\prime}$ and $\mathscr{R}\left(\mathscr{H}_{\alpha}, 1 \otimes \mathscr{B}\left(\mathbb{C}^{2}\right), \Omega_{\alpha}, \alpha \in \mathbb{N}\right)$. Thus, $\left\{W^{*} \widetilde{T}_{\alpha} W\right\}_{\alpha \in \mathbb{N}}$ provides the desired sequence.

2. (i) $\Rightarrow$ (iii): Let $\mathscr{M}$ (respectively $\mathscr{R}_{1}$ ) be represented with cyclic and separating vector in the Hilbert space $\mathscr{H}_{0}$ (respectively $\mathscr{H}_{1}$ ). Then the isomorphism $\mathscr{M} \simeq \mathscr{M} \otimes \mathscr{R}_{1}$ is spatial and there exists a unitary $W: \mathscr{H} \rightarrow \mathscr{H}_{0} \otimes \mathscr{H}_{1}$ intertwining $\mathscr{M}$ with $\mathscr{M} \otimes \mathscr{R}_{1}$ and $\mathscr{M}^{\prime}$ with $\left(\mathscr{M} \otimes \mathscr{R}_{1}\right)^{\prime}=\mathscr{M}^{\prime} \otimes \mathscr{R}_{1}^{\prime}$. Then pick sequences $\left\{A_{i, \alpha}\right\}_{\alpha \in \mathbb{N}}$, $\left\{B_{i, \alpha}\right\}_{\alpha \in \mathbb{N}}, i=1,2$, of the form $\left\{1 \otimes \widetilde{A}_{i, \alpha}\right\}_{\alpha \in \mathbb{N}},\left\{1 \otimes \mathscr{\mathscr { B }}_{i, \alpha}\right\}_{\alpha \in \mathbb{N}}$, with $\left\{\widetilde{A}_{i, \alpha}\right\}_{\alpha \in \mathbb{N}},\left\{\widetilde{B}_{i, \alpha}\right\}_{\alpha \in \mathbb{N}}$ chosen as in step 1 . The unitary equivalence already established and step 1 yield the claim.

3. (ii) $\Rightarrow$ (iii): Let $\Gamma$ denote the $\sigma$-weakly closed convex hull of the set of operators $T=\frac{1}{2}\left(A_{1}\left(B_{1}+B_{2}\right)+A_{2}\left(B_{1}-B_{2}\right)\right)$ with $A_{i} \in \mathscr{M}$ and $B_{i} \in \mathscr{M}^{\prime}$ admissible. $\Gamma$ is $\sigma$-weakly compact, since the unit sphere in $\mathscr{B}(\mathscr{H})$ is compact in the $\sigma(\mathscr{B}(\mathscr{H}), \mathscr{T}(\mathscr{H}))$-topology. Then for every $\phi$ as described in (ii) there exists such a $T \in \Gamma$ such that $\beta\left(\phi, \mathscr{M}, \mathscr{M}^{\prime}\right)=\phi(T)$. For any unit vector $\Phi \in \mathscr{H}$, let $\Gamma(\Phi)$ $\equiv\{T \in \Gamma \mid\langle\Phi, T \Phi\rangle=\sqrt{2}\}$. This is a $\sigma$-weakly closed convex face of $\Gamma$. Since by hypothesis

$$
\sqrt{2}=\beta\left(\frac{1}{n} \sum_{i=1}^{n}\left\langle\Phi_{i}, \cdot \Phi_{i}\right\rangle, \mathscr{M}, \mathscr{M}^{\prime}\right)=\sup _{T \in \Gamma} \sum_{i=1}^{n} \frac{1}{n}\left\langle\Phi_{i}, T \Phi_{i}\right\rangle,
$$

for any finite collection $\left\{\Phi_{i}\right\}_{i=1}^{n}$ of unit vectors, it follows that $\bigcap_{i=1}^{n} \Gamma\left(\Phi_{i}\right) \neq \emptyset$ for any such collection. Hence, $\bigcap_{\| \Phi=1} \Gamma(\Phi) \neq \emptyset$, by the compactness of $\Gamma$.

4. (iii) $\Rightarrow$ (ii): This implication is trivial.

5. (iii) $\Rightarrow$ (i): Set $A_{\alpha}=\frac{1}{2}\left(A_{1, \alpha}+i A_{2, \alpha}\right)$ and

$$
B_{\alpha}=\frac{1}{2 \sqrt{2}}\left(B_{1, \alpha}+B_{2, \alpha}+i\left(B_{1, \alpha}-B_{2, \alpha}\right)\right)
$$


$\alpha \in \mathbb{N}$. Then for any unit vector $\Omega \in \mathscr{H}$,

$$
\begin{aligned}
& \left\langle\Omega,\left(1-2^{-1 / 2} T_{\alpha}\right) \Omega\right\rangle=\left\|\left(A_{\alpha}-B_{\alpha}\right) \Omega\right\|^{2}+\left\|\left(A_{\alpha}^{*}-B_{\alpha}^{*}\right) \Omega\right\|^{2} \\
& \quad+\frac{1}{2}\left\langle\Omega,\left\{\left(1-A_{1, \alpha}^{2}\right)+\left(1-A_{2, \alpha}^{2}\right)+\left(1-B_{1, \alpha}^{2}\right)+\left(1-B_{2, \alpha}^{2}\right)\right\} \Omega\right\rangle \equiv \varepsilon_{\alpha}^{2}
\end{aligned}
$$

converges to zero as $\alpha \rightarrow \infty$ by hypothesis. Note $\left(1-A_{i, \alpha}^{2}\right)$ and $\left(1-B_{i, \alpha}^{2}\right)$ are positive. Hence, as $\alpha \rightarrow \infty$ the following quantities are of order $\varepsilon_{\alpha}$, where $\varepsilon_{\alpha} \downarrow 0$ :

$$
\left\|\left(A_{\alpha}-B_{\alpha}\right) \Omega\right\|,\left\|\left(A_{\alpha}^{*}-B_{\alpha}^{*}\right) \Omega\right\|,\left\|\left(1-A_{i, \alpha}^{2}\right) \Omega\right\|,\left\|\left(1-B_{i, \alpha}^{2}\right) \Omega\right\| .
$$

[Here one should recall that if $C \geqq 0,\|C\| \leqq 1$, and $\langle\Omega, C \Omega\rangle=O(\varepsilon)$, then $\left\|C^{1 / 2} \Omega\right\|^{2}=O(\varepsilon)$ and thus $\|C \Omega\|^{2}=O(\varepsilon)$.] For any $A \in \mathscr{M}$,

$$
\left|\left\langle\Omega,\left[A, A_{\alpha}\right] \Omega\right\rangle\right|=\left|\left\langle\Omega,\left[A, A_{\alpha}-B_{\alpha}\right] \Omega\right\rangle\right| \leqq\|A\| \cdot O\left(\varepsilon_{\alpha}\right) .
$$

Moreover, since $\left[A_{\alpha}, B_{\alpha}\right]=0$ all $\alpha \in \mathbb{N}, A_{\alpha}^{2}-B_{\alpha}^{2}=\left(A_{\alpha}-B_{\alpha}\right)\left(A_{\alpha}+B_{\alpha}\right)$, so that

$$
\left\|\left(A_{\alpha}^{2}-B_{\alpha}^{2}\right) \Omega\right\| \leqq\left\|\left(A_{\alpha}+B_{\alpha}\right)\right\|\left\|\left(A_{\alpha}-B_{\alpha}\right) \Omega\right\|=O\left(\varepsilon_{\alpha}\right) .
$$

Similarly,

$$
\left\|\left(A_{\alpha}^{* 2}-B_{\alpha}^{* 2}\right) \Omega\right\|=O\left(\varepsilon_{\alpha}\right) .
$$

Further, note that $B_{\alpha}^{2}-B_{\alpha}^{* 2}=i\left(B_{1, \alpha}^{2}-B_{2, \alpha}^{2}\right)$, so that

$$
\left\|\left(B_{\alpha}^{2}-B_{\alpha}^{* 2}\right) \Omega\right\| \leqq\left\|\left(1-B_{1, \alpha}^{2}\right) \Omega\right\|+\left\|\left(1-B_{2, \alpha}^{2}\right) \Omega\right\|=O\left(\varepsilon_{\alpha}\right) .
$$

Thus, by (2.4)-(2.6),

$$
\begin{aligned}
& \left\|\frac{1}{2}\left(A_{1, \alpha} A_{2, \alpha}+A_{2, \alpha} A_{1, \alpha}\right) \Omega\right\|=\left\|\left(A_{\alpha}^{2}-A_{\alpha}^{* 2}\right) \Omega\right\| \\
& \quad \leqq\left\|\left(A_{\alpha}^{2}-B_{\alpha}^{2}\right) \Omega\right\|+\left\|\left(B_{\alpha}^{2}-B_{\alpha}^{* 2}\right) \Omega\right\|+\left\|\left(B_{\alpha}^{* 2}-A_{\alpha}^{* 2}\right) \Omega\right\|=O\left(\varepsilon_{\alpha}\right) .
\end{aligned}
$$

Also, note that

$$
\begin{aligned}
& \left\|\left(A_{1, \alpha}-\frac{1}{\sqrt{2}}\left(B_{1, \alpha}+B_{2, \alpha}\right)\right) \Omega\right\|=\left\|\left(A_{\alpha}+A_{\alpha}^{*}-B_{\alpha}-B_{\alpha}^{*}\right) \Omega\right\| \\
& \quad \leqq\left\|\left(A_{\alpha}-B_{\alpha}\right) \Omega\right\|+\left\|\left(A_{\alpha}^{*}-B_{\alpha}^{*}\right) \Omega\right\|=O\left(\varepsilon_{\alpha}\right) .
\end{aligned}
$$

Therefore,

$$
\begin{aligned}
& \left\langle\Omega,\left(1-\left(\frac{i}{2}\left[A_{1, \alpha}, A_{2, \alpha}\right]\right)^{2}\right) \Omega\right\rangle=1-\left\|\frac{1}{2}\left[A_{1, \alpha}, A_{2, \alpha}\right] \Omega\right\|^{2} \\
& \quad=1-\left\langle\Omega, A_{1, \alpha} A_{2, \alpha} A_{2, \alpha} A_{1, \alpha} \Omega\right\rangle+O\left(\varepsilon_{\alpha}\right)
\end{aligned}
$$

[using (2.7)]

$$
=1-\left\langle\Omega, A_{1, \alpha} A_{2, \alpha}^{2} \frac{1}{\sqrt{2}}\left(B_{1, \alpha}+B_{2, \alpha}\right) \Omega\right\rangle+O\left(\varepsilon_{\alpha}\right)
$$

[by (2.8)]

$$
\begin{aligned}
& =1-\left\langle\frac{1}{\sqrt{2}}\left(B_{1, \alpha}+B_{2, \alpha}\right) \Omega, A_{1, \alpha} A_{2, \alpha}^{2} \Omega\right\rangle+O\left(\varepsilon_{\alpha}\right) \\
& =1-\left\langle A_{1, \alpha} \Omega, A_{1, \alpha} A_{2, \alpha}^{2} \Omega\right\rangle+O\left(\varepsilon_{\alpha}\right)
\end{aligned}
$$


[where (2.8) was used again]

$$
=1-\left\langle A_{1, \alpha}^{2} \Omega, A_{2, \alpha}^{2} \Omega\right\rangle+O\left(\varepsilon_{\alpha}\right)=O\left(\varepsilon_{\alpha}\right) .
$$

From (2.3) and Proposition 2.8 of [12] one can conclude that $\left\{A_{\alpha}\right\}_{\alpha \in \mathbb{N}}$ is a centralizing sequence and since $\mathscr{C}_{\omega}(\mathscr{M})$ is a $C^{*}$-algebra, $\left\{A_{1, \alpha}\right\}_{\alpha \in \mathbb{N}}$ and $\left\{A_{2, \alpha}\right\}_{\alpha \in \mathbb{N}}$ must also be centralizing sequences. Let $a_{1}, a_{2}$ be the corresponding elements in $\mathscr{M}_{\omega}(\mathscr{M})$ for some (any) ultrafilter $\omega$. By (2.9) and the positivity of $1-\left(\frac{i}{2}\left[A_{1, \alpha}, A_{2, \alpha}\right]\right)^{2}$, one has $\left(\frac{i}{2}\left[A_{1, \alpha}, A_{2, \alpha}\right]\right)^{2} \Omega$ converging strongly to $\Omega$ as $\alpha \rightarrow \infty$, for arbitrary $\Omega \in \mathscr{H}$. Thus, $\left(\frac{i}{2}\left[a_{1}, a_{2}\right]\right)^{2}=1$ in $\mathscr{M}_{\omega}(\mathscr{M})$, so that $\mathscr{M}_{\omega}(\mathscr{M})$ is not abelian. Theorem 2.2 then yields the condition (i) if $\mathscr{M}$ is factor.

If $\mathscr{M}$ is not a factor, let

$$
\mathscr{M}=\int^{\oplus} \mathscr{M}(\zeta) d v(\zeta), \quad \mathscr{H}=\int^{\oplus} \mathscr{H}(\zeta) d v(\zeta),
$$

be its central decomposition. Condition (iii) then holds in $v$-almost all $\mathscr{H}(\zeta)$ with the corresponding operators in the decompositions

$$
\begin{aligned}
A_{i, \alpha} & =\int^{\oplus} A_{i, \alpha}(\zeta) d v(\zeta), B_{j, \alpha}=\int^{\oplus} B_{j, \alpha}(\zeta) d v(\zeta), \\
T_{\alpha} & =\int^{\oplus} \frac{1}{2}\left(A_{1, \alpha}(\zeta)\right)\left(B_{1, \alpha}(\zeta)+B_{2, \alpha}(\zeta)\right)+A_{2, \alpha}(\zeta)\left(B_{1, \alpha}(\zeta)-B_{2, \alpha}(\zeta)\right) d v(\zeta) .
\end{aligned}
$$

It follows from the above demonstration that $\mathscr{M}(\zeta) \simeq \mathscr{M}(\zeta) \otimes \mathscr{R}_{1}$ for $v$-almost all $\mathscr{M}(\zeta)$. Thus, $\mathscr{M} \simeq \mathscr{M} \otimes \mathscr{R}_{1}$ (see Proposition 10 in Sect. II.3 of [35]).

Theorem 2.3 makes an unexpected connection between the generic maximal violation of Bell's inequalities, condition (ii), and the technical property of strong stability, condition (i). Moreover, if either of these conditions holds, then there exists a sequence of admissible quadruples that in the limit maximally violate Bell's inequalities in all normal states on $\mathscr{B}(\mathscr{H})$. We shall see in the next chapter that there are many physically interesting cases known where the algebras of local observables are in fact strongly stable, or type III $_{1}$ for the application of Theorem 2.1.

\section{Applications to Quantum Field Theory}

In this section we present the genericity of the maximal violation of Bell's inequalities as an application of the results established in the previous chapter. Let $\{\mathscr{A}(\mathcal{O})\}_{\mathcal{O} \subset \mathbb{R}^{4}}$ be a net of local von Neumann algebras in a separable Hilbert space $\mathscr{H}$ with the net satisfying the usual axioms of isotony, locality and Poincaré covariance under the action of a nontrivial, strongly continuous, unitary representation $U\left(\mathscr{P}_{+}^{\uparrow}\right)$ of (the covering group of) the Poincare group $\mathscr{P}_{+}^{\uparrow}$ that satisfies the spectrum condition (see, e.g. $[27,1]$ for further details about these axioms). The net is said to satisfy duality for the wedge algebras if $\mathscr{A}(W)=\mathscr{A}\left(W^{\prime}\right)^{\prime}$ for every wedge-shaped region $W \in \mathscr{W}$ (see, e.g. [22]). We mention that although we discuss here explicitly only nets of "observable algebras," all results are valid 
mutatis mutandis for nets of local field algebras satisfying the assumptions in [20]. Also, all results are valid for two, three and four space-time dimensions.

We present first an immediate consequence of Theorem 2.1 for algebraic QFT in the following corollary, and then we discuss conditions on the structure $\left(\mathscr{H},\{\mathscr{A}(\mathcal{O})\}, U\left(\mathscr{P}_{+}^{\uparrow}\right)\right)$ under which the hypothesis of the corollary are satisfied. Thereafter, Theorem 2.3 will be treated similarly.

Corollary 3.1. Let $\{\mathscr{A}(\mathcal{O})\}_{\mathcal{O} \subset \mathbb{R}^{4}}$ be a net of von Neumann algebras in a Hilbert space $\mathscr{H}$ as above that satisfies, in addition,

(i) $\mathscr{A}(W)$ is a type $I I I_{1}$ factor for each $W \in \mathscr{W}$,

(ii) $\mathscr{A}(W)=\mathscr{A}\left(W^{\prime}\right)^{\prime}$ for each $W \in \mathscr{W}$.

Then for each vector state $\phi$ on $\mathscr{B}(\mathscr{H}), \beta\left(\phi, \mathscr{A}(W), \mathscr{A}\left(W^{\prime}\right)\right)=\sqrt{2}$.

Remark. By the covariance assumption and the fact that every wedge region $W$ is the appropriate Poincaré transform of any given $W_{0} \in \mathscr{W}$, it suffices that (i) and (ii) be satisfied by one wedge algebra $\mathscr{A}(W)$.

Let $P_{0}$ denote the selfadjoint projection in $\mathscr{H}$ onto the subspace of $U\left(\mathscr{P}_{+}^{\uparrow}\right)$ invariant vectors. In [17] (see also [28]) it was shown that if the dimension of $P_{0} \mathscr{H}$ is one, then assumption (i) does indeed hold. In addition, in [9] it was shown that if $\left(\mathscr{H},\{\mathscr{A}(\mathcal{O})\}, U\left(\mathscr{P}_{+}^{\uparrow}\right)\right)$ is a massive single particle representation, i.e. if the mass hyperboloid $H_{m}=\left\{p \in \mathbb{R}^{4} \mid p^{2}=m^{2} ; p_{0}>0\right\}$ is contained in the singular spectrum of the generators of the translation subgroup of $U\left(\mathscr{P}_{+}^{\uparrow}\right)$ and if the joint spectrum of these generators is contained in $H_{m} \cup\left\{p \in \mathbb{R}^{4} \mid p^{2} \geqq M^{2}, p_{0}>0\right\}$ for some $M>m>0$, then there exists a (vacuum) representation $\left(\pi, \mathscr{H}_{\pi}\right)$ of $\mathscr{A}$ that is Poincare-covariant, for which $\operatorname{dim} P_{0} \mathscr{H}_{\pi}=1$, and for which the algebras associated to regions in the causal complement of some space-like cone are unitarily equivalent to the corresponding algebra in the original net (see [9] for details). Hence, if $\left(\mathscr{H},\{\mathscr{A}(\mathcal{O})\}, U\left(\mathscr{P}_{+}^{\uparrow}\right)\right)$ is a massive single particle representation, then condition (i) holds. The same is true, and for similar reasons, for the representations of the quasilocal observable algebras in the superselection sectors appearing in the theory of superselection structure of Doplicher et al. [16]. There may well be other physically interesting situations in which assumption (i) in Corollary 3.1 obtains. If so, these would provide natural candidates for maximal violation of Bell's inequalities in all pure normal states in the representation.

Without assumption (ii) one could conclude that $\beta\left(\phi, \mathscr{A}(W), \mathscr{A}(W)^{\prime}\right)=\sqrt{2}$ for all vector states, but unless (ii) holds, the violators in $\mathscr{A}(W)^{\prime}$ may well not be localized in the wedge $W^{\prime}$. Duality for the wedge algebras is known to be satisfied by the net $\{\mathscr{A}(\mathcal{O})\}$ if $\operatorname{dim} P_{0} \mathscr{H} \geqq 1$ and if there exists a quantum field $\varphi$ satisfying the standard axioms [30] with a cyclic vector $\Omega \in P_{0} \mathscr{H}$, and the field is associated with the net in the following weak sense:

(C) There exists a test function $f_{s}$ of compact support $\left[\operatorname{supp}\left(f_{s}\right) \subset \mathcal{O}_{s} \in \mathscr{K}\right.$, the set of double cones in $\mathbb{R}^{4}$ ] whose Fourier transform vanishes nowhere and satisfies

$$
\left\langle\varphi\left(f_{s}\right) \Phi, A \Psi\right\rangle=\left\langle A^{*} \Phi, \varphi\left(f_{s}\right)^{*} \Psi\right\rangle
$$

for all $A \in \mathscr{A}\left(\mathcal{O}_{s}\right)^{\prime}$ and all $\Phi, \Psi \in \mathscr{P}_{0, s}\left(\mathbb{R}^{4}\right) \Omega$, where $\mathscr{P}_{0, s}\left(\mathbb{R}^{4}\right)$ is the smallest unital *-algebra containing $\varphi\left(f_{s}\right)$ and all of its Poincaré transforms. 
For a proof of this claim and for further conditions that also yield assumption (ii), see [22]. An independent set of sufficient conditions using the funnel property and duality in another (locally normal) representation can also be put together using $[19,31]$.

Thus, assumptions (i) and (ii) of Corollary 3.1 hold in many situations of physical interest. The reader can formulate the obvious theorems for himself. We shall present a generalization of one such theorem that we mention here explicitly because it makes another point.

Theorem 3.2. Let $\{\mathscr{A}(\mathcal{O})\}$ be a net of local von Neumann algebras such that $(C)$ holds for a quantum field $\varphi$ satisfying the above-mentioned conditions. Then $\beta\left(\phi, \mathscr{A}(W), \mathscr{A}\left(W^{\prime}\right)\right)=\sqrt{2}$ for any $W \in \mathscr{W}$ and any vector state $\phi$ in $\mathscr{H}$.

Proof. The additional point here is that only $\operatorname{dim} P_{0} \mathscr{H} \geqq 1$ is assumed and not $\operatorname{dim} P_{0} \mathscr{H}=1$ as required to apply $[17,28]$. If $\operatorname{dim} P_{0} \mathscr{H}>1$, then the algebras $\{\mathscr{A}(W) \mid W \in \mathscr{W}\}$ are not factors [20]. But under the given assumptions it follows from [20-22] that the central decomposition of the quasilocal algebra $\mathscr{A}^{\prime \prime}=\left\{\mathscr{A}(\mathcal{O}) \mid \mathcal{O} \subset \mathbb{R}^{4}\right\}^{\prime \prime}$ leads to a direct integral decomposition of $\left(\mathscr{H},\{\mathscr{A}(\mathcal{O})\}, U\left(\mathscr{P}_{+}^{\uparrow}\right), \varphi(f), \Omega\right)$ into "pure phases" $\mathscr{H}(\zeta)$, etc., where $\operatorname{dim}\left(P_{0} \mathscr{H}\right)(\zeta)=1$ and in which $\mathscr{A}(W)(\zeta)$ is a type III $_{1}$ factor for all $W \in \mathscr{W}$ (see $[20,21]$ for details). Since this decomposition gives a standard probability measure $v$ on the spectrum of the center of $\mathscr{A}^{\prime \prime}$ such that

$$
\begin{aligned}
\phi & \left(A_{1}\left(B_{1}+B_{2}\right)+A_{2}\left(B_{1}-B_{2}\right)\right)=\left\langle\Phi,\left\{A_{1}\left(B_{1}+B_{2}\right)+A_{2}\left(B_{1}-B_{2}\right)\right\} \Phi\right\rangle \\
\quad & \int^{\oplus}\left\langle\Phi(\zeta),\left\{A_{1}(\zeta)\left(B_{1}(\zeta)+B_{2}(\zeta)\right)+A_{2}(\zeta)\left(B_{1}(\zeta)-B_{2}(\zeta)\right)\right\} \Phi(\zeta)\right\rangle d v(\zeta),
\end{aligned}
$$

and since by Corollary 3.1 and the above remarks

$$
\beta\left(\phi_{\zeta}, \mathscr{A}(W)(\zeta), \mathscr{A}\left(W^{\prime}\right)(\zeta)\right)=\sqrt{2}
$$

$v$-almost everywhere, the assertion of the theorem follows.

We now turn to applications of Theorem 2.3.

Theorem 3.3. Let one of the following obtain.

(i) $\{\mathscr{A}(\mathcal{O})\}_{\mathcal{O} \subset \mathbb{R}^{4}}$ is a net of local algebras associated to a Bose of Fermi free quantum field (in the sense $(C)$ ) with finitely many components in a separable Hilbert space $\mathscr{H}$. [The uniqueness of the (cyclic) vacuum is not required.]

(ii) $\{\mathscr{A}(\mathcal{O})\}_{\mathcal{O} \subset \mathbb{R}^{4}}$ is a net of local algebras in a vacuum representation with at least one vacuum vector $\Omega$ cyclic for $\mathscr{A}(\mathcal{O})$, all $\mathcal{O} \in \mathscr{K} \cup \mathscr{W}$. The net is locally generated, i.e. for each $W \in \mathscr{W}, \mathscr{A}(W)=\{\mathscr{A}(\mathcal{O}) \mid \mathcal{O} \in \mathscr{K}, \mathcal{O} \subset W\}^{\prime \prime}$ and for each $\mathcal{O} \in \mathscr{K}, \mathscr{A}(\mathcal{O})$ $=\left\{\mathscr{A}\left(\mathcal{O}_{0}\right) \mid \mathcal{O}_{0} \in \mathscr{K}, \mathcal{O}_{0} \subsetneq \mathcal{O}\right\}^{\prime \prime}$, it satisfies the funnel property, i.e. for any $\mathcal{O}_{1}, \mathcal{O}_{2} \in \mathscr{K}$ such that the closure of $\mathcal{O}_{1}$ is contained in the interior of $\mathcal{O}_{2}$ there exists a type I von Neumann algebra $\mathscr{M}$ such that $\mathscr{A}\left(\mathcal{O}_{1}\right) \subset \mathscr{M} \subset \mathscr{A}\left(\mathcal{O}_{2}\right)$, and it satisfies duality for wedges and double cones. Moreover, $\mathscr{A}(\mathcal{O})$ has no type $I I I_{0}$ summand, all $\mathcal{O} \in \mathscr{K}$.

Then for every normal state $\phi$ on $\mathscr{B}(\mathscr{H})$,

$$
\beta\left(\phi, \mathscr{A}(W), \mathscr{A}\left(W^{\prime}\right)\right)=\sqrt{2}=\beta\left(\phi, \mathscr{A}(\mathcal{O}), \mathscr{A}\left(\mathcal{O}^{\prime}\right)\right),
$$


for all $W \in \mathscr{W}$ and $\mathcal{O} \in \mathscr{K}$. (If $\{\mathscr{A}(\mathcal{O})\}$ is a net of Fermi field algebras, then $\mathscr{A}\left(W^{\prime}\right)$ and $\mathscr{A}\left(\mathcal{O}^{\prime}\right)$ in $(3.2)$ are replaced by their images under the appropriate Klein transform see $[16,20]$.)

Proof. (i) If $\mathcal{O} \in \mathscr{K} \cup \mathscr{W}$, then $\mathscr{A}(\mathcal{O})$ is an injective von Neumann algebra, since it is the weak closure of a CCR- or CAR-algebra over a separable Hilbert space. It is infinite because the vacuum vector is cyclic and separating for all $\mathscr{A}(\mathcal{O}), \mathcal{O} \in \mathscr{K} \cup \mathscr{W}$. But infinite, injective algebras are strongly stable [14, 2], excepting some type III $_{0}$ algebras; but $\mathscr{A}(\mathcal{O})$ is not type III $_{0}$ (it is type III $_{1}$ [25]). Theorem 2.3 yields the assertion at once, since duality holds for these algebras.

(ii) Since type I algebras are injective [14], it follows from Proposition 6.4 of [14] that $\mathscr{A}(\mathcal{O})$ is injective for each $\mathcal{O} \in \mathscr{K} \cup \mathscr{W}$. It is infinite by hypothesis and the reason given above. By the assumptions and $[14,2]$, it follows that it is strongly stable.

Remark. Fredenhagen [25] gives very general conditions that, in (ii), yield the assumption that double cone algebras have no type $\mathrm{III}_{0}$ summand as a consequence.

The assumptions (ii) are known to hold in a number of interesting examples $[19,31]$. And work is underway to prove more generally that wedge algebras are typically injective [23]. In the next chapter we discuss the above results and some possible extensions.

\section{Discussion}

We shall restrict ourselves here to discussing one point in particular - the remarkable genericity of maximal violation of Bell's inequalities in the sense that $\beta\left(\phi, \mathscr{A}(W), \mathscr{A}\left(W^{\prime}\right)\right)=\sqrt{2}$ for all normal states on $\mathscr{B}(\mathscr{H})$. It should be emphasized that maximal violation, or even violation of Bell's inequalities, is not generic in this sense in quantum mechanics. It was shown in Theorem 2.1 in [32] that if $\phi$ is a convex sum of product states across $\mathscr{A}(W)$ and $\mathscr{A}\left(W^{\prime}\right)$, then $\beta\left(\phi, \mathscr{A}(W), \mathscr{A}\left(W^{\prime}\right)\right)=1$, i.e. Bell's inequalities hold in such states. In quantum mechanics the algebras of observables are type I, and there are many normal product states across commuting type I algebras. Quantum field theory is thus even less compatible with "local hidden-variables" theories than quantum mechanics is (we refer the reader to [32] for a mathematically exact elucidation of what we mean by "local hidden-variables theory").

If $\mathcal{O}_{1}$ and $\mathcal{O}_{2}$ are strictly spacelike separated regions, then the genericity of maximal violation, even of violation, can fail. For example, when the net satisfies the funnel property, then there exist normal product states across $\mathscr{A}\left(\mathcal{O}_{1}\right)$ and $\mathscr{A}\left(\mathcal{O}_{2}\right)$. However, the algebras associated to tangent double cones, i.e. double cones whose closures intersect in only one point, will not have normal product states across them. We conjecture the genericity of maximal violation of Bell's inequalities across such algebras, and because such regions are of finite extent, it is of physical relevance to prove this conjecture. Work is underway on this problem. For a proof of maximal violation of Bell's inequalities across tangent double cones at least in the vacuum state in dilation-invariant theories, see $[33,32]$. 
Acknowledgements. The authors wish to thank Prof. John E. Roberts and Dr. Lothar Schmitt for bringing the paper [15] to their attention. Most of this work was carried out while SJS was visiting the Fachbereich Physik, Universität Osnabrück under partial support of a University of Rochester Faculty Research Grant. SJS wishes to thank his friends and colleagues in Osnabrück, particularly Helga Versace, for their warm hospitality.

\section{References}

1. Araki, H.: Local quantum theory, I. In: Local quantum theory. Jost, R. (ed.). New York: Academic Press 1969

2. Araki, H., Woods, E.J.: A classification of factors. Publ. Res. Inst. Math. Sci., Kyoto Univ. 4, 51-130 (1968)

3. Aspect, A., Grangier, P., Roger, G.: Experimental tests of realistic local theories via Bell's theorem. Phys. Rev. Lett. 47, 460-463 (1981)

4. Aspect, A., Grangier, P., Roger, G.: Experimental realization of Einstein-Podolsky-RosenBohm Gedankenexperiment: A new violation of Bell's inequalities. Phys. Rev. Lett. 49, 91-94 (1982)

5. Aspect, A., Dalibard, J., Roger, G.: Experimental test of Bell's inequalities using time-varying analyzers. Phys. Rev. Lett. 49, 1804-1807 (1982)

6. Bell, J.S.: On the Einstein-Podolsky-Rosen paradox. Physics 1, 195-200 (1964)

7. Bell, J.S.: On the problem of hidden variables in quantum mechanics. Rev. Mod. Phys. 38, 447-452 (1966)

8. Buchholz, D.: Product states for local algebras. Commun. Math. Phys. 36, 287-304 (1974)

9. Buchholz, D., Fredenhagen, K.: Locality and the structure of particle states. Commun. Math. Phys. 84, 1-54 (1982)

10. Clauser, J.F., Horne, M.A.: Experimental consequence of objective local theories. Phys. Rev. D. 10, 526-535 (1974). See also: Clauser, J.F., Horne, M.A., Shimony, A., Holt, R.A.: Proposed experiment to test local hidden-variable theories. Phys. Rev. Lett. 23, 880-884 (1969)

11. Connes, A.: Une classification des facteurs de type III. Ann. Sci. École Norm. Sup. 6, 133-252 (1973)

12. Connes, A.: Almost periodic states and factors of type III $_{1}$. J. Funct. Anal. 16, 415-445 (1974)

13. Connes, A.: Outer conjugacy classes of automorphisms of factors. Ann. Sci. École Norm. Sup. 8, 383-420 (1975)

14. Connes, A.: Classification of injective factors. Ann. Math. 104, 73-115 (1976)

15. Connes, A., Størmer, E.: Homogeneity of the state space of factors of type III $_{1}$. J. Funct. Anal. 28, 187-196 (1978)

16. Doplicher, S., Haag, R., Roberts, J.E.: Fields, observables, and gauge transformations, I, II. Commun. Math. Phys. 13, 1-23 (1969) and 15, 173-200 (1969)

17. Driessler, W.: Comments on lightlike translations and applications in relativistic quantum field theory. Commun. Math. Phys. 44, 133-141 (1975)

18. Driessler, W.: On the type of local algebras in quantum field theory. Commun. Math. Phys. 53, 295-297 (1977)

19. Driessler, W.: Duality and absence of locally generated superselection sectors for CCR-type algebras. Commun. Math. Phys. 70, 213-220 (1979)

20. Driessler, W., Summers, S.J.: Central decomposition of Poincaré-invariant nets of local field algebras and absence of spontaneous breaking of the Lorentz group. Ann. Inst. Henri Poincaré 43, 147-166 (1985)

21. Driessler, W., Summers, S.J.: On the decomposition of relativistic quantum field theories into pure phases. Helv. Phys. Acta 59, 331-348 (1986)

22. Driessler, W., Summers, S.J., Wichmann, E.H.: On the connection between quantum fields and von Neumann algebras of local operators. Commun. Math. Phys. 105, 49-84 (1986)

23. Driessler, W., Summers, S.J.: Work in progress

24. Fine, A.: Hidden variables, joint probability, and the Bell's inequalities. Phys. Rev. Lett. 48, 291-295 (1982) 
25. Fredenhagen, K.: On the modular structure of local algebras of observables. Commun. Math. Phys. 97, 79-89 (1985)

26. Garg, A., Mermin, N.D.: Farkas's lemma and the nature of reality: Statistical implications of quantum correlations. Found Phys. 14, 1-39 (1984)

27. Haag, R., Kastler, D.: An algebraic approach to quantum field theory. J. Math. Phys. 5, 848-861 (1964)

28. Longo, R.: Notes on algebraic invariants for non-commutative dynamical systems. Commun. Math. Phys. 69, 195-207 (1979)

29. Sakai, S.: $C^{*}$-algebras and $W^{*}$-algebras. Berlin, Heidelberg, New York: Springer 1971

30. Streater, R.F., Wightman, A.S.: PCT, Spin and statistics, and all that. New York: Benjamin 1964

31. Summers, S.J.: Normal product states for fermions and twisted duality for CCR- and CARtype algebras with application to the Yukawa ${ }_{2}$ quantum field model. Commun. Math. Phys. 86, 111-141 (1982)

32. Summers, S.J., Werner, R.: Bell's inequalities and quantum field theory. I. General setting. Preprint (submitted)

33. Summers, S.J., Werner, R.: Bell's inequalities and quantum field theory. II. Bell's inequalities are maximally violated in the vacuum. Preprint (submitted)

34. Takesaki, M.: Structure of factors and automorphism groups. Providence, RI: Am. Math. Soc. 1983

35. Dixmier, J.: Les algèbres d'opérateurs dans l'espace Hilbertien. Paris: Gauthier-Villars 1969

Communicated by K. Osterwalder

Received September 9, 1986 
